Meta

Journal des traducteurs

Translators' Journal

\title{
Contexte, compréhension, traduction
}

\section{Chen Wei}

Volume 44, numéro 1, mars 1999

Théorie et pratique de la traduction en Chine

The Theory and Practice of Translation in China

URI : https://id.erudit.org/iderudit/002225ar

DOI : https://doi.org/10.7202/002225ar

Aller au sommaire du numéro

\section{Éditeur(s)}

Les Presses de l'Université de Montréal

\section{ISSN}

0026-0452 (imprimé)

1492-1421 (numérique)

Découvrir la revue

\section{Citer cet article}

Wei, C. (1999). Contexte, compréhension, traduction. Meta, 44(1), 144-153.

https://doi.org/10.7202/002225ar

\section{Résumé de l'article}

Pour traduire, il faut d'abord comprendre. Maisÿÿomprendre quoi ? Il est loin d'être suffisant de comprendre uniquement les signifiés car, appartenant à la langue et faisant partie d'un ensemble structuré, les signifiés ne nous fournissent que des virtualités sémantiques. C'est plutôt le sens qu'il faut comprendre et traduire. Le sens est la base de la fidélité authentique d'une traduction. La compréhension du sens se fait généralement par une analyse des contextes : contexte verbal immédiat, qui aide à lever la polysémie des signes ; contexte verbal élargi, qui permet de désigner le sens d'un énoncé, et contexte situationnel, qui est indispensable pour saisir le vouloir-dire du traducteur. Ces contextes sont nécessaires pour la compréhension, mais aussi pour la traduction. Le traducteur doit donc dépasser la limite de la langue et effectuer ses analyses dans le domaine de la parole. 


\title{
Contexte, compréhension, traduction
}

\author{
chen wei \\ Shanghai International Studies University, \\ Shanghai, Chine
}

\begin{abstract}
RÉSUMÉ
Pour traduire, il faut d'abord comprendre. Mais comprendre quoi? II est loin d'être suffisant de comprendre uniquement les signifiés car, appartenant à la langue et faisant partie d'un ensemble structuré, les signifiés ne nous fournissent que des virtualités sémantiques. C'est plutôt le sens qu'il faut comprendre et traduire. Le sens est la base de la fidélité authentique d'une traduction. La compréhension du sens se fait généralement par une analyse des contextes: contexte verbal immédiat, qui aide à lever la polysémie des signes; contexte verbal élargi, qui permet de désigner le sens d'un énoncé, et contexte situationnel, qui est indispensable pour saisir le vouloir-dire du traducteur. Ces contextes sont nécessaires pour la compréhension, mais aussi pour la traduction. Le traducteur doit donc dépasser la limite de la langue et effectuer ses analyses dans le domaine de la parole.
\end{abstract}

\section{ABSTRACT}

Understanding is the first phase of the translation process. But what does the act of understanding entail? To understand is to extract meaning, contextual meaning (signification) not virtual meaning. Meaning provides the basis for fidelity in translation. Extracting meaning involves analyzing context and situation. To achieve this, the translator cannot confine himself to language per se, but must concern himself with language in use.

\section{INTRODUCTION}

La traduction, de même que toute opération de langue, s'opère à deux niveaux distincts mais complémentaires: celui du signifié et celui du sens. Le signifié d'un mot ou d'un énoncé et son sens ne se confondent pas ni ne se correspondent, le sens d'un énoncé n'est pas équivalent à la somme des signifiés qui composent son énonciation. Le signifié est une partie du contenu informationnel que revêt l'énoncé, et qui reste constante, indépendante des contextes et des situations dans lesquels cet énoncé est émis et reçu; le signifié appartient à l'énoncé en tant que réalisation d'un système sémique. Comme le signifié est un élément de la langue faisant partie d'un ensemble structuré, on pourrait en espérer une étude plus ou moins complète et précise. Alors que le sens, lui, échappe à l'inventaire et à la description sémantique, parce qu'il est la partie du contenu informationnel tributaire des contextes et des situations, et infiniment variable selon les coordonnées de ces contextes et de ces situations. Ainsi, le sens, mis en rapport avec les éléments extralinguistiques, est un fait de parole, distinct de la langue tout en étant fonction. La langue attribue un ou des signifiés aux mots et aux phrases, la parole les enrichit de notions inimaginables au seul plan lexicologique. 
On voit que le sens, loin d'être statique et donné objectivement, est un processus de déroulement constant qui se construit tout au long du discours. Et, dans les pages qui suivent, nous chercherons à traiter spécifiquement des éléments qui interviennent dans ce processus qui donne vie au sens, et des incidences que ces éléments pourraient porter à l'opération traduisante.

La linguistique moderne, dans son étude de la langue, a touché aux notions de contexte et de situation. Cela dit, il nous semble indispensable de scinder la notion de contexte en deux : contexte verbal immédiat et contexte verbal élargi (Lederer 1986).

En comparant des traductions à leurs originaux et en analysant la cause de leur réussite ou de leur échec, nous pouvons constater que le contexte verbal immédiat, le contexte verbal élargi et la situation (qu'on appelle aussi parfois contexte situationnel) constituent justement les trois facteurs sur lesquels s'appuie le cheminement du traducteur vers la compréhension du message original.

Le texte verbal immédiat est nécessai re pour limiter les virtualités sémantiques de la langue (ou d'un signe) ; le contexte verbal élargi permet de désigner le sens d'un énoncé, et il est aussi important pour l'univocité de l'information que l'est le contexte verbal immédiat pour l'univocité des signes; alors que la situation est capitale pour la compréhension du vouloir-dire (non explicité dans la plupart des cas) du locuteur.

Cette distinction faite, nous tenons à préciser que l'explication de ces trois facteurs est démonstrative pour une théorie de la traduction, car dans la communication, la compréhension s'accomplit normalement sans difficultés et en un laps de temps tellement court que l'on ne se rend même pas compte de sa complexité. C'est seulement en y prêtant volontairement attention que l'on arrive à illustrer le rôle de ces trois facteurs dans la compréhension.

\section{CONTEXTE VERBAL IMMÉDIAT}

Le contexte verbal immédiat correspond «à la capacité de la mémoire immédiate», c'est-à-dire à la «présence simultanée d'un ensemble de mots dans la mémoire», ou encore, dans le cas de la traduction écrite, «à l'empan de l'appréhension visuelle» (Lederer 1986: 44). L'étude du contexte verbal immédiat nous explique que la polysémie d'un mot est un état de langue et non un fait de parole. En effet, tout mot isolé hors contexte présente nombre de sens virtuels (c'est-à-dire nombre de signifiés), mais aucun sens réel. Cependant, une fois inséré dans un contexte verbal immédiat, la polysémie est tout de suite levée, et on saisit facilement l'acception pertinente des formes linguistiques en assemblage.

Par exemple, lorsque nous entendons prononcer la phrase: «Donnez-moi un morceau de /p $\widetilde{\varepsilon} /$ », nous ne faisons pas de choix entre le sens de «faire un cadeau » et de «tendre» pour comprendre le mot «donner»; nous n'avons pas à écarter le sens de «morceau de musique» pour retenir celui de «tranche», à propos de «morceau »; et nous n'évoquons pas non plus le sens de «pin » avant de saisir celui de «pain ». Cela, parce qu'avec un contexte verbal immédiat donné, aucune polysémie n'affecte le mot, aucun choix entre différentes acceptions ne s'offre à l'auditeur ou au locuteur.

Dans la plupart des cas de l'opération traduisante, comme un minimum de contexte verbal immédiat est toujours fourni, on peut considérer que la polysémie du mot ne pose pas de vrai problème à la traduction, et qu'il est en conséquence extrê mement rare que le sens des mots ne soit pas univoque. 
Par exemple, chaque langue possède des mots dont la désignation varie d'un contexte à l'autre. Ce n'est donc qu'en examinant le contexte verbal immédiat qu'on arrive à déterminer leur sens actualisé.

(1) Texte original en chinois (exemple tiré de Yue Yang-lie 1987):

美国（读者文摘）认为，临睡前做睡眠呼息对失眠者特别有效。它的简易做法是:

(它 : /ta/, pronom, signifie «il» ou «elle»)

traduction en français:

Selon le Reader's Digest des États-Unis, faire des exercices de respiration avant de se coucher est un remède particulièrement efficace pour ceux qui souffrent d'insomnie. Ces exercices sont décrits ainsi...

(2) Texte original en chinois (exemple tiré de Yue Yang-lie 1987):

在纪念中国工农红军二万五千里长征五十周年之际，本报记者罗开富同志沿着红军 长征的路线, 开始进行徒步采访。这是一项很有意义的活动。

(这 : /zhel, déictique, signifie «ce», «ceci »)

traduction en français:

À l'occasion du 50 anniversaire de la Longue Marche de 25000 lis par l'Armée Rouge des ouvriers et des paysans de Chine, notre reporter Luo Kai-fu s'en va mener une enquête en suivant à pied le même itinéraire que celui emprunté autrefois par l'Armée Rouge. Ce voyage est une entreprise très significative.

(3) Texte original en chinois (exemple tiré de Yue Yang-lie 1987):

邮电部门抓本部门队伍的思想建设、作风建设、组织建设问题。

(抓 : /zhual, saisir, arrêter (qqn.); attirer (l'attention) ; accorder une importance à) traduction française:

Le ministre des Postes et Télégraphes accorde une attention particulière à la formation idéologique du personnel, à l'amélioration de son style de travail et à la sélection des candidats du Parti.

Le contexte verbal élargi peut aussi nous aider à déterminer la signification de certains syntagmes très usités, mais qui ne sont pas encore considérés comme des expressions toutes faites. Dans ces syntagmes, les mots connaissent souvent un «glissement de sens».

(4) Texte original en chinois (exemple tiré de Yue Yang-lie 1987):

实现了周总理生前宏愿，不再怕人在这一重要战略物资上卡脖子。

(卡㭙子 : /ka bo zi/, serrer le cou à qqn., étrangler)

traduction en français:

... le noble vœu formulé par le premier ministre Zhou de son vivant s'est enfin réalisé, et la Chine n'a plus à craindre qu'on la fasse chanter avec cette matière d'importance stratégique. 
(5) Texte original en chinois (exemple tiré de Yue Yang-lie 1987):

一方面是人口增长快，一方面是我国 底子薄，显然，不控制人口的增长，就不能促 进四个现代化的实现。

(底子 : /dizi/, la base, le fondement; 薄 : /bo/, mince)

traduction en français:

De cette croissance démographique très rapide, ajoutée au fait que la Chine a peu de richesse accumulée, il s'ensuit que sans un contrôle des naissances, la réalisation des quatre modernisations s'en ressentira.

(6) Texte original en chinois (exemple tiré de Yue Yang-lie 1987):

反对不关心群众痛㾕的官僚主义。( “毛选” 第五卷, p. 183)

(痛痒 : /tong yang/, douleur et chatouillement)

traduction en français:

Lutter contre la bureaucratie qui se désintéresse du bien-être des masses. (Euvres choisies de Mao Tsé tong, p. 202.)

De tous ces exemples cités, on pourrait tirer la conclusion que la majorité des mots assemblés dans le discours perdent leur caractère polysémique, et ceci n'est possible que lorsqu'ils sont insérés dans un certain contexte verbal immédiat. Ainsi, un traducteur ne doit jamais agir à la première vue d'un mot, car une traduction mot à mot sans tenir compte des contextes verbaux n'aboutirait pas à exprimer le vrai sens du mot.

\section{CONTEXTE VERBAL ÉLARGI}

À la différence du contexte verbal immédiat, le contexte verbal élargi est l'ensemble dynamique des informations qu'apporte au locuteur le déroulement du discours; il correspond à l'idée qui se dégage peu à peu du discours. Égal à zéro aux premiers mots du discours, le contexte verbal élargi gonfle de plus en plus au fil de l'énonciation et avec ce gonflement du contexte, l'auditeur ou le lecteur arrive peu à peu à lever les ambiguïtés des phrases du texte et à en construire un sens qui se succède. En effet, dans la lecture, nous n'avons ni le besoin, ni la possibilité, de retenir tous les mots; par contre, nous retenons ce que nous venons de lire suffisamment longtemps en mémoire cognitive pour comprendre la suite du texte. Par conséquent, de même que le contexte verbal immédiat fait surgir d'un mot un sens actualisé, et laisse dans l'ombre ses autres acceptions, de même le contexte verbal élargi refoule certains traits sémantiques de l'acception des mots dégagés par le contexte verbal immédiat. Voilà ce qui explique pourquoi un traducteur expérimenté prend toujours la peine de lire un texte intégralement avant d'en commencer la traduction.

Un exemple négatif nous montrera, de manière bien convaincante, que la compréhension de l'original nécessite un contexte plus large que la phrase et que, tout comme la traduction mot pour mot, la traduction décontextualisée n'aboutit pas à un texte authentiquement fidèle à l'original. 
(7) Texte original en français (exemple tiré de Luo Guo-lin 1981: 9-10) :

- Un nouveau? interroge le sergent qui vient d'entrer.

- Oui, répond le gendarme. Voilà le papier pour les vêtements.

- Bon. M ais vous les ramenez à la pelle.

- Sergent, on n'a jamais vu ça. Plus on en arrête, plus il y en a.

Le sergent haussa les épaules. Hilarion le regardait sournoisement. On lui tendit le gros pantalon et la veste à rayures blanches et bleues.

Traduction (I) :

“是个新来的吗? ”班长刚进来便这样问。

“是的”，宪兵回答。“那儿是包衣服的纸。”

“好。您带来的衣服可真多呀! ”

“班长，我从来就没见过。被捕的人越多，这种东西也就越多。”

班长的肩头笪了一下。依拉利翁偷偷地打量着他。有人给他一条宽大的长裤和一件 蓝白条的粗线上衣。

À première vue, cette traduction semble acceptable, car en la comparant phrase par phrase avec l'original, on dirait qu'il existe une équivalence presque totale. $\mathrm{Ce}$ pendant, nous n'oublions pas que le traducteur doit faire passer en langue cible non seulement les acceptions virtuelles que possède chaque phrase du texte, mais le sens du texte dégagé d'un contexte. Et c'est justement cela que notre traducteur a négligé. En fait, s'il avait bien fait son analyse contextuelle en étudiant intégralement le texte original, il se serait rendu compte qu'il s'agit en l'occurrence d'un dialogue entre un gendarme qui venait de ramener en prison un certain nommé Hilarion, et un sergent qui s'occupait de la distribution de vêtements aux prisonniers. II aurait aussi été averti que le papier que le gendarme présentait au sergent était un papier écrit en échange duquel celui-ci devait fournir un «uniforme» à son nouveau pensionnaire; que c'est toujours ce sergent, et non pas une autre personne, qui a tendu à Hilarion le gros pantalon et la vareuse; et qu'enfin, ladite vareuse n'est pas nécessairement une veste de grosse toile, mais une veste assez simple.

Le contexte original verbal explicité, nous pourrons comparer la traduction I à la traduction correcte pour voir combien un simple remplacement de phrase par phrase peut conduire un texte traduit loin de son original.

Traduction (II) :

“是个新来的吗? ”班长进来后问道。

“是的”，宪兵回答。“这是领衣服的条子。”

“好的。你们抓来的人可真多呀!”

“俺从来没见过这种事儿。班长，越抓反而越多。”

班长䇯了䇯肩。依拉利翁偷偷地打量着他。他给了依拉利翁一条肥大的裤子和一件

带有蓝白条纹的上衣。 
Après avoir examiné l'exemple ci-dessus, il est clair pour nous qu'une traduction sur le plan des phrases isolées présente les mêmes risques d'ambiguïté que celle du mot à mot. Si on traduit un texte phrase par phrase en s'inspirant plus de la langue originale que du continuum de la pensée de l'écrivain, on juxtapose des éléments linguistiques isolés qui se correspondent individuellement d'une langue à l'autre, mais qui, assemblés, représentent un puzzle mal ajusté à la forme naturelle que prendrait la pensée dans les autres langues. La traduction ne peut et ne doit s'effectuer qu'en considérant les contextes, et c'est seulement ainsi qu'on parvient à une vraie fidélité de sens.

\section{SITUATION (CONTEXTE SITUATIONNEL)}

La situation représente tous les éléments de perception sensorielle non linguistique, concomitants au discours. Elle est l'ensemble des informations explicites fournies par le discours. Plus clairement, elle est le cadre matériel, la salle où l'on se trouve, la vue que l'on a, les gestes et les mimiques de l'orateur, elle est aussi l'émetteur et le destinataire de l'énoncé... Lorsque la situation n'est pas pertinente, elle peut parfois distraire l'attention; Iorsqu'au contraire elle l'est, elle oriente la compréhension de l'énoncé linguistique vers le sens voulu par le locuteur.

Un exemple, fourni par Marouzeau (1963: 147), pourrait nous aider à mieux comprendre la notion de situation: «On demande, en se faufilant dans la presse pour parvenir à la sortie d'un wagon de métro. - Vous descendez, monsieur? - Non, mais je descendrai. »

Pris hors situation, l'énoncé en italique est incomplet, et donc ambigu. Or, dans la bouche d'un voyageur qui parle à un autre voyageur dans le même wagon de métro, cette phrase est parfaitement claire. II faudrait l'entendre par : «Ce n'est pas ici que je descends normalement, mais je descendrai pour dégager la sortie, quitte à remonter ensuite. » La situation fait le sens.

Voici un second exemple, donné par Lederer (1986: 44) :

«La lumière, s'il vous plaît ! » disait un congressiste qui présentait des diapositives au cours de sa communication. Lorsque ces mots résonnaient dans la salle obscure, le technicien allumait les lumières; lorsque quelques minutes après, le congressiste prononçait les mêmes mots, le technicien plongeait la salle dans le noir.

Par les mêmes mots connus, identiques, ce congressiste donnait successivement deux ordres diamétralement opposés, il était chaque fois compris, la perception d'une situation différente s'intégrant à un seul et mêmeénoncélinguistique pour constituer tantôt une idée, tantôt une autre. En l'absence de la situation, le mot «lumière» ne serait que convention linguistique.

Cela dit, la situation, qui confère un sens au discours et conditionne son énonciation, se laisse elle-même réduire à une analyse selon quatre paramètres:

- I'objet, ce dont il est question dans le message;

- l'émetteur, la personne qui énonce des mots ou des phrases;

- le récepteur, la ou les personnes auxquelles le message s'adresse;

- le vecteur, les conditions spatiales et temporelles dans lesquelles le message est énoncé.

Ces quatre paramètres conditionnent le message non seulement à sa production, mais aussi à sa réception. 
a) Voyons d'abord les indices de ces quatre paramètres sur la production du message.

Le fait que l'énonciation consiste à dégager, au moyen des mots considérés comme les plus adéquats, ce dont on parle (soit l'objet du message), est déjà vu comme un axiome. De ce point de vue, l'énonciation est en fin de compte une appropriation des termes à l'objet désigné. Aussi pourrons-nous dire que l'objet est le premier élément qui conditionne l'énonciation du message.

Mais il s'en faut de beaucoup que l'adaptation à l'objet soit le seul facteur conditionnant cette appropriation des termes à l'énoncé. L'émetteur, le destinataire, le vecteur jouent tous leur rôle dans la communication.

Soit les phrases:

1) Jean a fait fondre un peu de sel dans un verre d'eau.

2) Le chimiste a fait fondre un peu de sel dans un verre d'eau.

3) Fais fondre un peu de sel dans un verre d'eau !

4) Faites dissoudre 10 grammes de chlorure de sodium dans 10 centilitres d'eau !

5) Faire dissoudre 10 grammes de sel dans un verre d'eau.

Les cinq phrases traitent du même objet, mais celui-ci est énoncé différemment. Ceci parce que l'énoncé tire aussi sa forme de l'émetteur et le manifeste en même temps. L'émetteur énonce en fonction de ce qu'il est lui-même, et de la relation entre lui et le destinataire. Dans les phrases 1 et 2, par exemple, «Jean » et «le chimiste» peuvent fort probablement être une même personne, mais le fait que, dans les deux phrases, les appellations sont différentes manifeste clairement quel'émetteur de la phrase 1 est un intime de Jean et que ce n'est pas le cas pour celui de la phrase 2. L'émetteur conditionne donc l'énonciation du message à sa propre façon.

Voyons maintenant les phrases 3 et 4 . Si l'on se demande pourquoi ces deux phrases ne sont pas réductibles l'une à l'autre, puisque l'objet désigné est le même, il est tout à fait normal de prétendre que la phrase 3 est prononcée par une ménagère et la phrase 4 par un chimiste. Mais supposons cette fois que l'émetteur soit une seule personne. Alors à quoi attribuer la différence entre ces deux phrases? Bien évidemment à la différence des destinataires: «Fais fondre un peu de sel dans l'eau !», c'est le chimiste qui parle dans sa cuisine et qui s'adresse à sa femme; alors que lorsqu'il prononce «Faites dissoudre 10 grammes de chlorure de sodium dans 10 centilitres d'eau !», il est dans son laboratoire, devant tous ses élèves. La comparaison des phrases 3 et 4 et l'analyse de la situation nous font voir les influences que le destinataire du message peut exercer sur l'énonciation.

Mais l'énonciation du message est aussi fonction des conditions de temps et d'espace (c'est-à-dire des vecteurs) qui lui sont imparties. L'émetteur adapte toujours son énonciation aux moyens de transmission qui s'interposent entre lui et le destinataire. Comparons les phrases 3 et 5 , et nous verrons que, si l'on suppose que la phrase 3 est adressée de vive voix par une femme à son mari en situation partagée (par exemple, le couple est en train de préparer le repas dans la cuisine), on ne peut s'empêcher de dire que la phrase 5 est extraite d'une recette de cuisine dans un livre. Si la situation dans laquelle la femme prononce la phrase 3 est tout à fait partagée par son mari, celle de la phrase 5 ne l'est que partiellement: le destinataire devient impersonnel, c'est pourquoi l'émetteur du livre de recette a, lui aussi, utilisé une tournure impersonnelle (l'infinitif du verbe «faire»). 
À une telle explication du rôle que jouent les quatre paramètres de la situation dans la production de l'énoncé, il faut ajouter que l'importance de ces quatre paramètres est susceptible de varier considérablement selon les types de messages, et que les incidences d'un paramètre sur l'autre peuvent être plus ou moins grandes. Cependant, on ne saurait concevoir de message linguistique indépendant desdits quatre paramètres.

b) De même que l'adaptation aux quatre paramètres de la situation conditionne l'énonciation, de même la référence conditionne la réception et la compréhension de ce même message.

La compréhension d'un énoncé nécessite en effet que tous les termes soient référés correctement aux éléments de la situation qui y a donné naissance et qui lui confère son sens. Autrement dit, l'auditeur ou le lecteur doit savoir qui parle, à qui on parle, de quoi on parle, où, quand et comment on parle. Sans ces références, l'énoncé est ambigu pour le récepteur.

En prenant l'exemple de la phrase 2, il importe de savoir si le chimiste est à table ou dans son laboratoire, car selon les cas, le sens de «sel » ne sera pas le même: si le chimiste est à table, le «sel » ne sera qu'une espèce d'assaisonnement, alors que s'il est dans son laboratoire, le «sel » pourrait désigner «tous les composés dans lesquels I'hydrogène d'un acide a été remplacé par un métal ».

Le sens d'un message est donc bien autre chose que la simple addition des signifiés. Sauf exception, les énoncés linguistiques sont toujours susceptibles d'une compréhension variable selon les conditions dans lesquelles ils sont émis. Parmi les virtualités sémantiques du signifié, la référence aux paramètres situationnels sélectionne celles qui sont effectivement voulues par l'émetteur.

c) Envisageons donc maintenant l'importance de la situation, non plus seulement en fonction de la compréhension du message, mais en fonction de sa traduction. Pour ce faire, étudions un exemple.

Soit la phrase: «Je vais à l'école. » Derrière cette phrase simple, se cachent des sens multiples - et diverses possibilités de traduction - en fonction des paramètres de son émission. Considérons d'abord la compréhension de cet énoncé:

- Si c'est un petit garçon qui le prononce sur le trottoir, son cartable sur le dos, en réponse à la question «O ù vas-tu ?» d'un interlocuteur de rencontre, cet énoncé a donc pour sens: «Je suis en train de me diriger vers l'endroit où je recevrai de l'éducation. »

- Si c'est aussi un petit garçon qui le prononce, mais cette fois, au lieu d'être dans la rue, il est sur une plage, au bord de la mer, en maillot de bain et répond à l'intention d'un des amis de ses parents, dans ce cas, on comprendra que l'enfant entend par "Je vais à l'école»: «Je suis en âge d'aller à l'école» ou «Je suis scolarisé».

- Si c'est le père du petit garçon qui est l'émetteur de cette phrase, vêtu d'un costume et conduisant sa voiture et qui répond à la question «Où vas-tu?», il y a peu de chance pour que le sens de l'énoncé soit réduit à celui du premier cas. II est fort possible que le père veuille dire: «Je vais à l'école de mon fils pour voir son professeur.»

Le sens de cette phrase n'est nullement codé d'avance, de manière univoque, et ne peut être saisi que par relation avec les paramètres situationnels. Et cette multiplicité d'interprétations de l'énoncé rejaillit bien entendu sur la traduction. 
- Dans la première situation, la traduction sera: “我去上学” ou “我上学去”.

- Dans la deuxième situation, elle sera: “我在念书”.

- Dans la troisième situation, elle sera: “我去学校办事”.

Aucune traduction en chinois énumérée ci-dessus n'est interchangeable avec les autres. Leur adéquation comme équivalent de traduction du message dépend des différents paramètres de la situation dans laquelle le message est énoncé.

Un autre exemple montrera que la traduction mot à mot peut laisser entendre un sens différent du sens voulu par le locuteur, et que la considération de la situation peut corriger la faute causée par le transcodage (Lederer 1986: 50-51).

En septembre 1973, le Président de la France, Georges Pompidou, a effectué une visite officielle en Chine. Avant son départ, on a remplacé le slogan «Chaleureuse bienvenue au Président Pompidou » par cet autre: «Saluons chaleureusement le dé part de Monsieur Pompidou », ce qui est visiblement une traduction mot à mot de l'expression chinoise “热烈欢送蓬皮杜先生." M ais cette traduction était un peu ambiguë, car elle équivalait aussi à se réjouir du départ du visiteur. Heureusement, les journalistes français se sont rendu compte de la contradiction évidente entre l'énoncé linguistique et la vraie intention que l'hôte voulait exprimer. C'est grâce à leurs remarques que l'on a remplacé à nouveau le slogan par «Chaleureux au revoir au Président Pompidou!»

\section{CONCLUSION}

L'étude que nous avons effectuée dans les pages précédentes sur les divers facteurs qui conditionnent le cheminement du sujet vers la compréhension du sens du discours nous montre que, dans la traduction, on a toujours affaire à deux types de «sens»: celui que présente la phrase isolée et celui que présente l'énoncé intégré dans des contextes et dans des situations. Dans le premier cas, la phrase est interprétée en fonction de connaissances dont on dispose de façon générale: on comprend quelque chose, ne serait-ce à l'extrême limite que le signifié en langue; mais cette compréhension n'est jamais qu'une hypothèse sur le sens, et la traduction qu'on peut en donner n'est somme toute qu'une hypothèse, donc pas forcément fidèle à l'original, et pour cause. Dans le second cas, celui d'une phrase intégrée dans des contextes et des situations données, la compréhension de l'énoncé s'appuie sur des facteurs extralinguistiques et s'effectue par l'exclusion spontanée de tous les «sens» virtuels non pertinents de la phrase.

De ce fait, un traducteur doit tenir compte non seulement des signifiés des mots, mais aussi de ces éléments extra-linguistiques qui conditionnent la compréhension. D'autant plus qu'en situation normale de communication, comme les interlocuteurs sont toujours en condition de situation plus ou moins partagée, le locuteur n'énonce jamais tout ce qu'il veut faire comprendre, il ne dit que le non-connu; c'est au récepteur de compléter lui-même le message à l'aide de la situation partagée. Plus la situation partagée est grande, moins il est nécessaire que le locuteur soit explicite dans son énonciation.

Ainsi, nous retournons à la thèse répétée cent mille fois par les traducteurs aussi bien que par les théoriciens de la traduction, et qui est pourtant souvent négligée par 
beaucoup de traducteurs débutants: une traduction mot à mot, ou phrase par phrase, n'aboutira pas à une fidélité authentique, laquelle doit être recherchée au plan du sens, et pour ce faire, il faut que le traducteur dépasse le domaine de la langue pour effectuer ses analyses dans celui des contextes et de la situation.

\section{RÉFÉRENCES}

Leder er, M. (1986) : «Implicite et explicite», dans Interpréter pour traduire, Paris, Didier Érudition.

Luo, Guo-lin (1981) : Théorie et techniques de la traduction du français en chinois, Beijing.

M a r o uzeau, J. (1963) : Précis de stylistique française, Paris, Masson.

M o un in, G. (1955) : Les Belles Infidèles, Marseille, Cahiers du Sud.

- - (1976) : Linguistique et traduction, Bruxelles, Dessart et Mardaga.

Per gnier, M. (1980) : Les Fondements sociolinguistiques de la traduction, Paris, Champion.

Sel eskovit ch, D. et M. Leder er (dir.) (1986) : Interpréter pour traduire, Paris, Didier Érudition. Van oye, F. (1973) : Expression, communication, Paris, Armand Colin.

Yuan, Ting-hong (1986) : Identité de la paroleà travers la différence des langues: de la traductibilité du chinois en français et vice versa.

Yue, Yang-lie (1987) : Exercices de traduction du chinois au français, Shanghai. 